\title{
Magnetic Field Effect on an Electron Tunneling out of a
} Confining Plane

\author{
Ping Ao \\ Department of Physics, FM-15 \\ University of Washington, Seattle, WA 98195
}

December 16, 2018

\begin{abstract}
The influence of a magnetic field on the tunneling of an electron out of a confining plane is studied by a path integral method. We map this 3 -d problem on to a 1 -d one, and find that the tunneling is strongly affected by the field. Without a perpendicular field the tunneling at zero temperature can be completely suppressed by a large parallel field, but in the small parallel field and low temperature limit the tunneling rate is finite. An explicit formula is obtained in this case. A quantitative explanation without fitting parameter to a recent experiment is provided.
\end{abstract}

PACS\#s: 73.40.Gk; 73.20.Dx; 
The tunneling of charged particles in the presence of a magnetic field has many peculiar properties. It occurs in the study of the escape of trapped electrons from a helium-vacuum interface 11, 2, 3], the focus of the present paper. It is, however, a general phenomenon and is widely encountered in mesoscopic systems [4], in semiconductor heterostructures [5, 6], in disorder media[7], and in Quantum Hall Systems such as the tunneling between double layers [8] and the quasiparticle tunneling[9]. The helium-vacuum-interface system is relatively simple, but it shares many features of the systems in Refs. [4, 5, 6, 7, 8, 9], such as the influences of magnitude and direction of a magnetic field and of many-body correlations on the tunneling. This system provides us a well controlled test ground for our understanding of tunneling in higher dimensions. There is a considerable experimental study of the escape rate on it [1, 3]. In the case of no magnetic field the tunneling process in this system is adequately understood, which is effectively a 1-d problem even after the consideration of many-body correlations[1], 2]. However, the presence of a magnetic field requires a full 3-d study of the tunneling process. It gives rise to new effects about the magnetic field and temperature dependence of the tunneling rate, as shown in a recent experiment which requires a clear and detailed theoretical explanation[3]. The purpose of the present paper is to give a formulation of the problem in this system and to present a detailed analytical study. A quantitative agreement without fitting parameter with the experiment of Ref. [3] is obtained. Our results, as well as our path integral method which keeps the relevant degrees of freedom but eliminates irrelevant ones by integration, may have a wider applications in the systems of Refs. [4, 5, 6, 7, 8, 9]. In fact, our Hamiltonian is identical to those, for example, in the study of magnetotransportation in semiconductor heterostructures[5].

Our main results are as follows. We consider a situation in 3-d that in one direction an electron has a metastable state, and there is no force in the remaining two directions. The tunneling out the metastable state is studied as a function of both magnitude and direction 
of an externally applied constant magnetic field, and of temperature. We map this problem onto that of quantum dissipative tunneling. The in-plane motion acts as an environment to the tunneling in the perpendicular direction because of the parallel magnetic field. This environmental influence is found to be subohmic when the perpendicular component of the magnetic field $B_{\perp}=0$, and superohmic when $B_{\perp} \neq 0$. From the obtained effective action we find that the tunneling is strongly influenced by the magnetic field. In particular, if $B_{\perp}=0$ and the magnetic field $B_{\|}$parallel to the force free plane is large enough there is a complete suppression of tunneling rate at zero temperature, and the electron becomes dynamically localized. However, if $B_{\|}$is small, the tunneling rate out of the metastable state is finite. Perturbatively, the semiclassical action is proportional to $B_{\|}^{2}$, when $B_{\perp}=0$. In this case the calculation can be generalized to a finite but low temperature, where we find that the semiclassical action decreases linearly with temperature $T$. The last two results are in a remarkable agreement with the experiment of Ref. [3].

Now we present the calculation leading to the above results. For the convenience of calculation we shall first assume that the $x$ - and $y$-directions have weak harmonic potentials. We take them to be zero, that is, $\omega_{0} \rightarrow 0$, in the end of the calculation. The Hamiltonian for an electron with mass $m$ and charge $e$ is then

$$
H=\frac{1}{2 m}\left[\mathbf{P}-\frac{e}{c} \mathbf{A}(\mathbf{R})\right]^{2}+\frac{1}{2} m \omega_{0}^{2}\left(x^{2}+y^{2}\right)+V(z)
$$

with the vector potential $\mathbf{A}$ determined by $\nabla \times \mathbf{A}=\mathbf{B}$. Here the metastable point of the potential is taken at $z=0$. For large enough $z, V(z)=-F z$, and there is a barrier separating this region and $z=0$. The external magnetic field is tilted: $\mathbf{B}=\left(B_{\|}, 0, B_{\perp}\right)$. In accordance with the calculation of tunneling, the vector potential will be taken as

$$
\mathbf{A}=\left(0, B_{\perp} x-B_{\|} z, 0\right)
$$

and it can be shown that results are independent of the choice of gauge because of the 
periodic boundary condition in the calculation of the tunneling rate. This is a $3-\mathrm{d}$ tunneling problem because the magnetic field couples the motions in all directions.

The tunneling is described by the Euclidean action 10, 11

$$
S[\mathbf{R}(\tau)]=\int_{0}^{\hbar \beta} d \tau\left[\frac{1}{2} m \dot{\mathbf{R}}^{2}+i \frac{e}{c}\left(B_{\perp} x-B_{\|} z\right) \dot{y}+V(z)+\frac{1}{2} m \omega_{0}^{2}\left(x^{2}+y^{2}\right)\right],
$$

where $\beta=1 / k_{B} T$ is the inverse temperature. The tunneling rate is proportional to $\exp \left\{-S_{c} / \hbar\right\}$, where the semiclassical action $S_{c}$ is determined by the bounce solution of the equation $\delta S=0$, in which the periodic boundary condition, $\mathbf{R}(\hbar \beta)=\mathbf{R}(0)$, is required. This is so-called the method of the imaginary part of the free energy $F=-k_{B} T \ln Z$ [12 calculated from the partition function

$$
Z=\int d \mathbf{R}^{\prime} D\left(\mathbf{R}^{\prime} ; \mathbf{R}^{\prime}\right)
$$

with the density matrix

$$
D\left(\mathbf{R}^{\prime}(0) ; \mathbf{R}^{\prime \prime}(\hbar \beta)\right)=\int \mathcal{D} \mathbf{R} \exp \left\{-\frac{1}{\hbar} S[\mathbf{R}]\right\}
$$

This method is identical to the WKB method at zero temperature, and allows us to have a unified treatment of the escape rate for finite temperatures.

We are interested in the electron tunneling out of the metastable state $z=0$ in the $z$-direction. After the tunneling other degrees of freedoms, $x$ and $y$, can take any allowed value. Therefore the summation over final states, integrations over the $x$ and $y$ coordinates, will be taken in the calculation of the partition function. Those are gaussian integrals. To integrate over the $x$-coordinate, we perform a Fourier transformation on the time interval $[0, \hbar \beta]:$

$$
(x(\tau), y(\tau), z(\tau))=\frac{1}{\hbar \beta} \sum_{n=-\infty}^{\infty}\left(x_{n}, y_{n}, z_{n}\right) e^{i \nu_{n} \tau} .
$$

Here $\nu_{n}=2 \pi n / \hbar \beta$. The action $S$ of eq.(3) can be then rewritten as

$$
S\left[\left\{\mathbf{R}_{n}\right\}\right]=\frac{1}{\hbar \beta} \sum_{n=-\infty}^{\infty}\left[\frac{1}{2} m \nu_{n}^{2} z_{n} z_{-n}+V(z)_{n}+\frac{1}{2}\left(m \nu_{n}^{2}+m \omega_{0}^{2}\right) x_{n} x_{-n}\right]
$$




$$
+\frac{1}{\hbar \beta} \sum_{n=-\infty}^{\infty}\left[\frac{1}{2}\left(m \nu_{n}^{2}+m \omega_{0}^{2}\right) y_{n} y_{-n}+\frac{e}{c}\left(B_{\perp} x_{n}-B_{\|} z_{n}\right) \nu_{-n} y_{-n}\right] .
$$

Here $\nu_{n}=-\nu_{-n}$ has been used. Now, we can shift the origin of $\left\{y_{n}\right\}$, and integrate over them. Then we obtain the effective action as

$$
\begin{gathered}
S_{e f f}\left[\left\{x_{n}, z_{n}\right\}\right]=\frac{1}{\hbar \beta} \sum_{n=-\infty}^{\infty}\left[\frac{1}{2} m \nu_{n}^{2} z_{n} z_{-n}+V(z)_{n}+\frac{1}{2}\left(m \nu_{n}^{2}+m \omega_{0}^{2}\right) x_{n} x_{-n}\right] \\
-\frac{1}{\hbar \beta} \sum_{n=-\infty}^{\infty} \frac{1}{2} \frac{\left(\frac{e}{c}\right)^{2} \nu_{n} \nu_{-n}}{m \nu_{n}^{2}+m \omega_{0}^{2}}\left(B_{\perp} x_{n}-B_{\|} z_{n}\right)\left(B_{\perp} x_{-n}-B_{\|} z_{-n}\right) .
\end{gathered}
$$

Similarly, the integration over $\left\{x_{n}\right\}$, can be performed. The resulting effective action is

$$
\begin{aligned}
S_{e f f}[z(\tau)]=\int_{0}^{\hbar \beta} d \tau & {\left[\frac{1}{2} m \dot{z}^{2}+V(z)\right]-\frac{1}{2} \int_{0}^{\hbar \beta} d \tau \int_{0}^{\hbar \beta} d \tau^{\prime}\left[\bar{g}_{1}\left(\tau-\tau^{\prime}\right)\right.} \\
& \left.-g_{2}\left(\tau-\tau^{\prime}\right)\right]\left[z(\tau)-z\left(\tau^{\prime}\right)\right]^{2}
\end{aligned}
$$

with the first kernel

$$
\bar{g}_{1}(\tau)=\frac{1}{\hbar \beta} \sum_{n=-\infty}^{\infty} \frac{1}{2} \frac{\left(\frac{e}{c} B_{\|}\right)^{2} \nu_{n}^{2}}{m \nu_{n}^{2}+m \omega_{0}^{2}} e^{i \nu_{n} \tau}
$$

and the second kernel

$$
g_{2}(\tau)=\frac{1}{\hbar \beta} \sum_{n=-\infty}^{\infty} \frac{1}{2} \frac{\left[B_{\perp} B_{\|}\left(\frac{e}{c}\right)^{2} \nu_{n} \nu_{-n}\right]^{2}}{\left[m \nu_{n}^{2}+m \omega_{0}^{2}\right]\left[\left(\frac{e}{c} B_{\perp}\right)^{2} \nu_{n}^{2}+\left(m \nu_{n}^{2}+m \omega_{0}^{2}\right)^{2}\right]} e^{i \nu_{n} \tau} .
$$

Here $\int_{0}^{\hbar \beta} d \tau \bar{g}_{1}(\tau)=0$ and $\int_{0}^{\hbar \beta} d \tau g_{2}(\tau)=0$ have been used in arriving above result.

Eqs.(9-11) can be simplified. We note that the kernel $\bar{g}_{1}$ can be separated into two parts:

$$
\bar{g}_{1}(\tau)=\frac{1}{\hbar \beta} \frac{m}{2}\left(\frac{e B_{\|}}{m c}\right)^{2} \sum_{n=-\infty}^{\infty} e^{i \nu_{n} \tau}-g_{1}(\tau)
$$

with

$$
g_{1}(\tau)=\frac{m}{2}\left(\frac{e B_{\|}}{m c}\right)^{2} \frac{\omega_{0}}{2} \frac{\cosh \left[\omega_{0}\left(\frac{\hbar \beta}{2}-\tau\right)\right]}{\sinh \left[\frac{\hbar \beta \omega_{0}}{2}\right]} .
$$

The first part of the right hand of eq.(12) is simply a periodic delta function, which gives no contribution to the effective action and will be dropped. We further simplify the expressions by taking the limit of $\omega_{0}=0$, and find that

$$
\bar{g}_{1}(\tau)=-g_{1}(\tau)=-\frac{1}{\hbar \beta} \frac{m}{2}\left(\frac{e B_{\|}}{m c}\right)^{2}
$$


and

$$
g_{2}(\tau)=\frac{m}{2}\left(\frac{e B_{\|}}{m c}\right)^{2}\left[\frac{\omega_{\perp}}{2} \frac{\cosh \left[\omega_{\perp}\left(\frac{\hbar \beta}{2}-\tau\right)\right]}{\sinh \left[\frac{\hbar \beta \omega_{\perp}}{2}\right]}-\frac{1}{\hbar \beta}\right]
$$

with $\omega_{\perp}=B_{\perp} e / m c$. Then using eqs. $(14,15,9)$ we obtain the effective action for our problem as

$$
\begin{aligned}
S_{e f f}[z(\tau)]= & \int_{0}^{\hbar \beta} d \tau\left[\frac{1}{2} m \dot{z}^{2}+V(z)\right]+\frac{1}{2} \int_{0}^{\hbar \beta} d \tau \int_{0}^{\hbar \beta} d \tau^{\prime} \frac{m}{2}\left(\frac{e B_{\|}}{m c}\right)^{2} \times \\
& \frac{\omega_{\perp}}{2} \frac{\cosh \left[\omega_{\perp}\left(\frac{\hbar \beta}{2}-\left|\tau-\tau^{\prime}\right|\right)\right]}{\sinh \left[\frac{\hbar \beta \omega_{\perp}}{2}\right]}\left[z(\tau)-z\left(\tau^{\prime}\right)\right]^{2} .
\end{aligned}
$$

Thus, we have mapped the original 3-d problem onto a 1-d one. This is because the inplane motion, $x$ - and $y$-directions, effectively behaves as a bath for $z$-direction motion [10], where the parallel magnetic field $B_{\|}$serves as a coupling coefficient. This form of effective action is easy to handle because of the existence of a sophisticated technique to calculate the dissipative tunneling rate[10]. In terms of the dissipative tunneling [10], the environmental effect in the eq.(16) is a superohmic damping of $s=\infty$ for $B_{\perp} \neq 0$, where the environment consists of the in-plane cyclotron motion. If $B_{\perp}=0$, it is then a subohmic damping of $s=0$, where the environment consists of in-plane plane-waves. We should point out that no approximation is used in obtaining eq.(16). Simply by inspection of eq.(16), one can conclude that the effective bath due to the in-plane motion will influence the tunneling rate out of the confining plane by affecting the semiclassical action. A numerical calculation of the tunneling rate will be needed to cover whole parameter region. In the following we take $B_{\perp}=0$, and focus on the peculiar subohmic damping case with $B_{\|} \neq 0$.

From eq.(16), the effective action with $B_{\perp}=0$ is

$$
S_{e f f}[z(\tau)]=\int_{0}^{\hbar \beta} d \tau\left[\frac{1}{2} m \dot{z}^{2}+V(z)\right]+\frac{m}{4}\left(\frac{e B_{\|}}{m c}\right)^{2} \frac{1}{\hbar \beta} \int_{0}^{\hbar \beta} d \tau \int_{0}^{\hbar \beta} d \tau^{\prime}\left[z(\tau)-z\left(\tau^{\prime}\right)\right]^{2} .
$$

This equation is explicitly gauge invariant under the change $y \rightarrow y+$ constant, because of the periodic boundary condition of $x, y$ imposed in the tunneling calculation as pointed out 
above. If there is a tunneling solution, it can be shown that

$$
\lim _{\hbar \beta \rightarrow \infty} \int_{0}^{\hbar \beta} d \tau z(\tau)=\text { constant }
$$

for the semiclassical solution. This is the result of the period boundary condition that $\lim _{\hbar \beta \rightarrow \infty} z(\hbar \beta)=z(0)=0$ and the finiteness of the semiclassical action. Therefore, the effective action at zero temperature may be written as

$$
S_{e f f}[z(\tau)]=\int_{-\infty}^{\infty} d \tau\left[\frac{1}{2} m \dot{z}^{2}(\tau)+V(z(\tau))+\frac{m}{2}\left(\frac{e B_{\|}}{m c}\right)^{2} z^{2}(\tau)\right] .
$$

It shows that the original potential $V(z)$ is renormalized to $V(z)+m\left(e B_{\|} / m c\right)^{2} z^{2} / 2$, and the metastable state at $z=0$ becomes more stable. For a large $z$, the renormalized potential is positive now, and we have a double-well like potential 13. If the second local potential minimum is lower than the one at $z=0$, tunneling out of $z=0$ is finite. However, for a large enough parallel magnetic field $B_{\|}$, the second local potential minumum will be higher than the one at $z=0$. The tunneling rate is then zero. In this case, eq.(18) is not valid. This analysis suggests that there is a magnetic field induced localization transition at zero temperature. The violation of eq.(18) may serve as the indication for this transition, or alternatively, the comparison of the local two potential minima of the renormalized potential in eq.(19) may determine the critical magnetic field. Thus we have obtained that the $s=0$ dissipative environment is marginal for localization in tunneling from a metastable state, compared to the $s=1$ case for the tunneling splitting 14, 15.

Away from the localization region, for a small parallel magnetic field $B_{\|}$the tunneling rate is finite. In particular, for a very small parallel magnetic field and low temperatures the semiclassical action may be evaluated perturbatively:

$$
\begin{gathered}
S_{c}=\int_{-\infty}^{\infty} d \tau\left[\frac{1}{2} m \dot{z}_{c}^{2}(\tau)+V\left(z_{c}(\tau)\right)\right]+\frac{m}{2}\left(\frac{e B_{\|}}{m c}\right)^{2}\left[\int_{-\infty}^{\infty} d \tau z_{c}^{2}(\tau)\right. \\
\left.-\frac{k_{B} T}{\hbar}\left(\int_{-\infty}^{\infty} d \tau z_{c}(\tau)\right)^{2}\right]
\end{gathered}
$$


where $z_{c}(\tau)$ is the bounce solution at zero temperature without the magnetic field, determined by the equation $m \dot{z}_{c}^{2} / 2=V\left(z_{c}\right)$. Eq.(20) shows a pronounced $B_{\|}^{2}$ dependence and linear temperature dependence.

Now we briefly discuss the effect of finite perpendicular magnetic field $B_{\perp}$. For $B_{\perp} \neq 0$ the effective bath changes from $s=0$ at $B_{\perp}=0$ to $s=\infty$. From Refs. [15, 12] we expect that the tunneling can occur at any value of $B_{\|}$and there is no localization. Therefore by tilting the direction of the magnetic field there should be a large change in the tunneling rate.

To conclude, we discsuss the experimental verification of the above results. The tunneling rate of trapped electrons escaping from a helium-vacuum interface was measured in Ref. [3] as a function of $B_{\|}^{2}$ and $T$ with $B_{\perp}=0$. A numerical estimation shows the data are in the low parallel magnetic field and low temperature limit. The comparison between the calculation according to eq.(20) and the data of Ref.[3] is shown in the Figure. A quantitative agreement is found in the low temperature limit. Note that there is no fitting parameter. The deviation in the high temperature end in the Figure suggests that the thermal activation starts to play a role. It is interesting to note that the corresponding critical magnetic field according to the renormalized potential in eq.(19) is about 1.3 Tesla for that experiment, which seems easy to realize experimentally. In Ref.[3] it is also found that there is no dependence of tunneling rate on $B_{\perp}$ when $B_{\|}=0$. This is implied in eq.(16), too, where by taking $B_{\|}=0$ the in-plane motion is decoupled from the tunneling in $z$-direction. To further assure oneself of the agreement between eq.(20) and the experimental data, the absence of the effect of the other dynamical correlations in the experiment need to be addressed. We discuss this below.

It has been shown that the response of the remaining 2-d electrons to the tunneling electron is superohmic [2]. The influence from ripplons is not only superohmic, also weak. The only possible ohmic effect is from the He atom scatterings. However, its strength is expo- 
nentially small at low temperature, and can be ignored at low temperatures. Because the superohmic bath has a weak influence on tunneling in the absence of a magnetic field[15, 12], the tunneling is therefore dominated by the adiabatic potential. Therefore the adiabatic potential is good in the explanation of the experiments [1] and dynamical correlations from those environments are weak[2]. Furthermore, it has been shown recently that, the super-

ohmic bath has a weak influence on the tunneling rate even in the presence of a magnetic field[16]. Therefore the adiabatic potential can be used in the tunneling rate calculation with a magnetic field, too. Using the adiabatic potential first proposed by Iye et al in Ref. [1], we have calculated the semiclassical action by eq.(20) as shown in the Figure. In the calculation the Stark shift and the distance of the 2-d electron layer from the helium-vacuum interface have been considered.

Acknowledgements: The assistance from Yong Tan on numerical calculation is appreciated. This work was supported by US National Science Foundation under Grant Nos DMR-8916052 and DMR-9220733.

\section{References}

[1] Y. Iye et al., J. Low Temp. Phys. 38, 293 (1980); K. Kono et al., ibid, 46, 195 (1982); E.Y. Andrei et al., Phys. Rev. Lett. 67, 3704 (1991); G.F. Saville et al., ibid, 70, 1517 (1993); and the references cited therein.

[2] P. Ao, Ph.D. thesis, University of Illinois at Urbana-Champaign, 1991 (unpublished); Mod, Phys. Lett. B7, 927 (1993); Physica B, in print.

[3] L. Menna et al., Phys. Rev. Lett. 70, 2154 (1993). 
[4] Mesoscopic Phenomena in Solids, Edited by B.L. Altshuler et al., North-Holland, Amsterdam, 1991.

[5] Physics of Low-Dimensional Semiconductor Structures, Edited by P. Butcher et al., Plenum, New York, 1993.

[6] P. Streda and A.H. MacDonald, Phys. Rev. B41, 11892 (1990); Y. Tan, ibid, 49, 1827 (1994).

[7] E. Medina and M. Kardar, Phys. Rev. B46, 9984 (1992).

[8] For exmaple, see, L. Zheng and A.H. MacDonald, Phys. Rev. B47, 10619 (1993); S. He, P.M. PLatzman, and B.I. Halperin, Phys. Rev. Lett. 71, 777 (1993).

[9] J.K. Jain et al., Phys. Rev. Lett. 71, 3003 (1993).

[10] A.O. Caldeira and A.J. Leggett, Ann. Phys.(NY), 149, 374 (1983); 153, 445(E) (1984).

[11] J.K. Jain and S. Kivelson, Phys. Rev. B37, 4111 (1988); H.A. Fertig and B.I. Halperin, ibid, B36, 7969 (1987).

[12] P. Hanggi et al., Rev. Mod. Phys. 62, 251 (1990).

[13] S.K. Bhattacharya and A.R.P. Rau, Phys. Rev. A26, 2315 (1982).

[14] S. Chakaravarty, Phys. Rev. Lett. 49, 681 (1982); A.J. Bray and M.A. Moore, ibid, 49, 1545 (1982); A. Schimd, ibid, 51, 1506 (1983); V. Hakim et al., Phys. Rev. B30, 464 (1984).

[15] A.J. Leggett et al., Rev. Mod. Phys. 59, 1 (1987).

[16] P. Ao and D.J. Thouless, Phys. Rev. Lett. 72, 132 (1994). 


\section{Figure Caption.}

Figure. The circles are the experimental data from Ref.[3]. The solid line is the calculation according to eq.(20), in which the 2-d electron density $n=8.7 \times 10^{7} / \mathrm{cm}^{2}$ and the external electric field $E=30 \mathrm{~V} / \mathrm{cm}$ are used. 